1998-7

\title{
Development and Experimental Validation of an Underwater Manipulator Hydrodynamic Model
}

Timothy W. McLain

Brigham Young University - Provo, mclain@byu.edu

Stephen M. Rock

Stanford University, rock@sun-valley.stanford.edu

Follow this and additional works at: https://scholarsarchive.byu.edu/facpub

Part of the Aerospace Engineering Commons, and the Mechanical Engineering Commons

\section{Original Publication Citation}

McLain, T. and Rock, S. Development and Experimental Validation of an Underwater Manipulator Hydrodynamic Model, International Journal of Robotics Research, vol. 17, no. 7, pp. 748-759, July 1998.

\section{BYU ScholarsArchive Citation}

McLain, Timothy W. and Rock, Stephen M., "Development and Experimental Validation of an Underwater Manipulator Hydrodynamic Model" (1998). Faculty Publications. 1521.

https://scholarsarchive.byu.edu/facpub/1521

This Peer-Reviewed Article is brought to you for free and open access by BYU ScholarsArchive. It has been accepted for inclusion in Faculty Publications by an authorized administrator of BYU ScholarsArchive. For more information, please contact ellen_amatangelo@byu.edu. 


\title{
Development and Experimental Validation of an Underwater Manipulator Hydrodynamic Model
}

\author{
Timothy W. McLain* Stephen M. Rock ${ }^{\dagger}$
}

\begin{abstract}
Hydrodynamic forces can be large and hence have a significant effect on the dynamic performance of underwater manipulation systems. This paper investigates these forces for a cylindrical single-link arm undergoing motions that are characteristic of a robotic manipulator. Based on flow visualization, theoretical analysis, and experimental measurements, a new model is developed that describes these forces. This model differs from previous models in that the drag and added-mass coefficients are statedependent functions that depend on the distance traveled by the arm. A factor of four improvement in accuracy is demonstrated over standard constant-coefficient models.
\end{abstract}

\section{Introduction}

For motions typical of an underwater manipulator, the hydrodynamic forces generated can be large and can significantly impact the dynamics of the system. This is especially true if a high performance (i.e., fast) manipulator is mounted on a relatively small vehicle platform. (The effect of these forces can be small for large vehicles with slowly moving manipulators.) However, though potentially important, relatively little is known about the hydrodynamic forces generated during these types of motions. A significant amount of literature is available that documents research on flow over cylindrical bodies (e.g., [Obasaju et al., 1988, Sarpkaya, 1992]), but most of it focuses on steady and sinusoidal two-dimensional flow past a stationary body, or on steady and sinusoidal motion of an infinitely long body in a stationary flow. Neither of these conditions accurately represents the flow typical of a robotic manipulator.

The unique attributes of manipulators and their motion require further attention: (1) the motion of robotic links typically has a large rotational component that induces complex three-dimensional flows; (2) robotic links rapidly accelerate and decelerate and seldom move at a constant velocity; (3) robotic motions are often limited to relatively small angles with resulting hydrodynamic forces never reaching steady state; and (4) robotic links have relatively small length-to-diameter ratios $(5<L / D<15)$ and exposed tips, which further complicate the flow.

Although some work relevant to robotic motion has been done, it fails to address adequately the complex nature of the flow over a manipulator and is consequently limited in its achievable accuracy. Lévesque and Richard [Lévesque and Richard, 1994], McMillan, Orin, and McGhee [McMillan et al., 1995], and Tarn, Shoults, and Yang [Tarn et al., 1996] have each addressed the modeling of underwater robotic systems, focusing on the efficient simulation of systems with many degrees of freedom. Their models are each striptheory-based and recommend using standard constant values for the drag and added-mass coefficients.

Developed here is a model that more accurately describes the hydrodynamic forces acting on a segment (and hence the whole) of an underwater robot arm. It is of the form

$$
d F=-C_{m}(s / D) \cdot \rho \frac{\pi D^{2}}{4} \dot{U} d l-C_{d}(s / D) \cdot \frac{1}{2} \rho D|U| U d l .
$$

where $d F$ is the hydrodynamic force generated on an arm segment, $C_{m}$ and $C_{d}$ are the added mass and the drag coefficients, $s$ is the distance traveled, $D$ is the diameter of the arm, $\rho$ is the water density, $U$ is the velocity of the arm, and $d l$ is the length of the arm segment.

\footnotetext{
*Department of Mechanical Engineering; 242 CB, Brigham Young University; Provo, UT 84602; tmclain@et.byu.edu

†Department of Aeronautics and Astronautics; 250 Durand Building, Stanford University; Stanford, CA 94305; rock@sun-valley.stanford.edu
} 
The important difference between this model and those previously used is that the drag and addedmass coefficients are functions of $s / D$. This theoretical result, an extension of the work done by Sarpkaya [Sarpkaya, 1963], is supported by results from flow visualization experiments. Furthermore, the shapes of the $C_{m}(s / D)$ and $C_{d}(s / D)$ curves hypothesized from the flow visualization experiment results are validated through parameter identification using torque and angle time-history measurements. Experimental results validate this approach.

\section{Experimental Apparatus}

For this research on the hydrodynamics of underwater arms, a circular cylinder swinging in a plane about one end was identified as the starting point because it represents a challenging, yet manageable problem that can be studied at a fundamental level. A single-link, single-degree-of-freedom arm shares many of the unique hydrodynamic attributes of a multiple-link, multiple-degree-of-freedom arm. Further, the axisymmetric shape of a cylinder of circular cross-section makes it well-suited for an underwater manipulator because hydrodynamic forces are independent of the orientation of the arm cross-section with respect to the flow, and the circular cross-section is a convenient geometry for water-proof housings for motors, electronics, and sensors.

The arm used was a $7.1 \mathrm{~cm}$ diameter, $0.65 \mathrm{~m}$ long hollow PVC cylinder mounted in a $2.3 \mathrm{~m}$ diameter by $1.5 \mathrm{~m}$ deep tank filled with water. The arm and its associated sensors are illustrated schematically in Figure 1. The arm was mounted so that it rotated about one end in a swinging motion. The actuator used to move the arm was a $1 / 2$ horsepower $(373 \mathrm{~W})$ variable-reluctance motor driving a 60:1 harmonic-drive reducer. The motor and harmonic drive were contained in a waterproof housing that was pressure-compensated with compressed air.

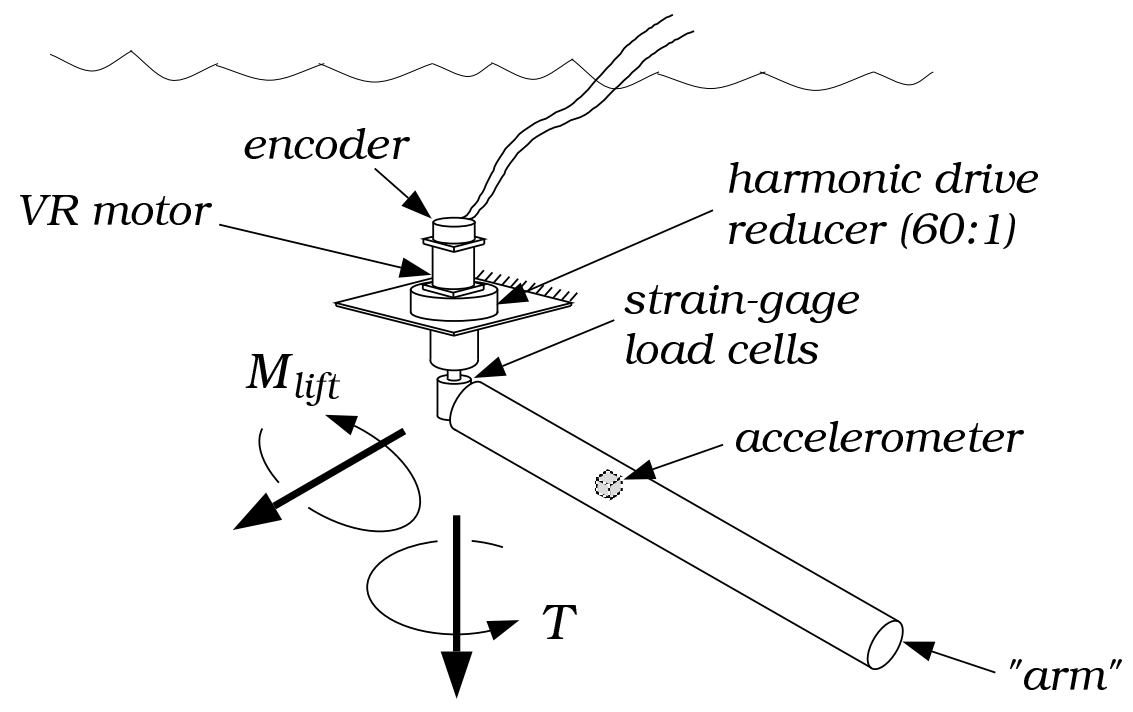

Figure 1: Schematic Diagram of Single-Link Arm, Actuator, and Sensors

To help characterize the hydrodynamic forces, several different types of sensors were used. Arm position was measured at $500 \mathrm{~Hz}$ using a 4096-count optical encoder. An estimate of the arm angular velocity was obtained by post-processing the position signal: The velocity was calculated by taking the first-difference of the position data and then filtering it forward and backward with a $25 \mathrm{~Hz}$ fourth-order Butterworth low-pass filter to reduce the noise without introducing undesirable phase lag.

To measure the angular acceleration of the arm, a linear solid-state accelerometer was mounted in the arm at a known distance from its hub. The accelerometer was mounted with its sensitive axis normal to the arm's longitudinal axis and in the horizontal plane to which the arm's motion was confined.

The output-shaft torque and lift moment at the arm hub were measured with a specially designed beam 
that connects the output shaft to the arm's base. This beam element has two full strain-gage bridges that measure both the output shaft torque $(T)$ due to inertial and in-line hydrodynamic forces and the lift moment $\left(M_{\text {lift }}\right)$ due to transverse forces generated from the shedding of vortices.

Flow visualization results were obtained by applying small amounts of a highly concentrated paste of food-grade dye to the cylinder at different locations and letting it dry in place. The arm was then submerged and videotaped while moving through specified slews. As the arm moved, the dye applied to the arm surface dissolved into the surrounding water, leaving a visual image of the water's motion. Video was taken from a top view with a camera looking down on the plane of arm motion, and also from a down-the-arm view with a camera attached to the arm at the hub looking out toward the tip.

\section{Hydrodynamic Model Development}

For a submerged body, such as a manipulator or vehicle, the net hydrodynamic force is due to the pressure distribution surrounding the body. This total force is commonly decomposed into two terms: in-line forces, which act in a direction collinear with the direction of motion; and transverse forces, which act in a direction normal to the direction of motion. Figure 2 shows a schematic representation of this simple decomposition. In-line forces are composed generally of the drag, added-mass, and fluid-acceleration forces; and transverse forces are due to lift from vortex shedding. (Note: if the cylinder and fluid accelerations are not collinear, this simple decomposition breaks down.)

Except for very slow motions, in-line forces are always significant for underwater manipulators. The significance of transverse forces is highly dependent on the occurrence of lock-in. Lock-in occurs when the vortex-shedding frequency coincides, for a sustained period of time, with a lightly-damped resonant mode of the system. When this happens, the shedding of vortices along the length of the arm becomes correlated, which results in oscillatory lift forces of a similar magnitude to that of the in-line forces. For typical robotic motions, lock-in does not occur because of the relatively short duration of the motions. In these situations, the magnitude of the transverse force is usually less than ten percent of that of the in-line force. The models in this paper only address the more common and dominant in-line forces. A more complete discussion of transverse forces and the conditions under which lock-in can occur for manipulators can be found in [McLain, 1995]. General discussions of drag, added-mass, fluid-acceleration, and lift forces as they pertain to manipulators can be found in [McMillan et al., 1995].

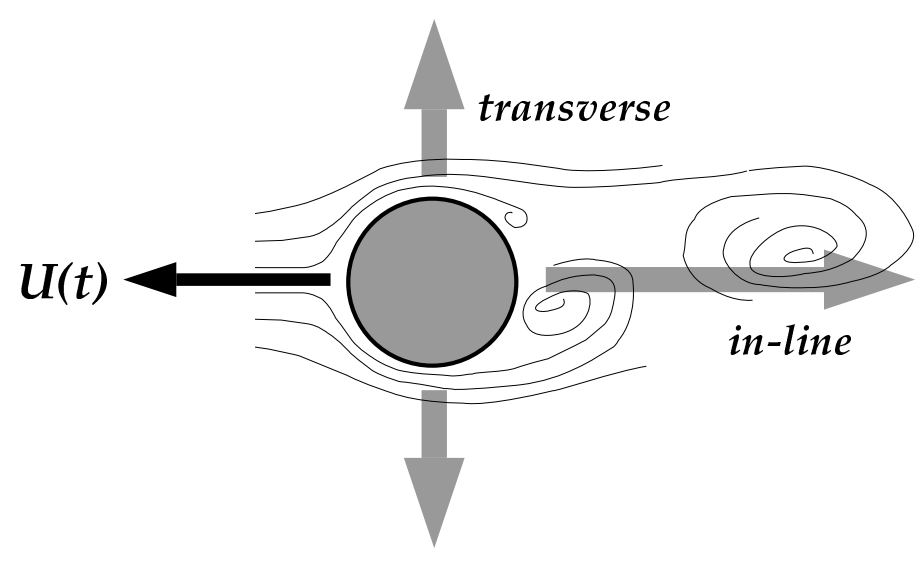

Figure 2: In-line and Transverse Hydrodynamic Forces

Models that describe these forces can be generated using several methods. For example, since the flow and pressure distribution around a body can be described by the Navier-Stokes equations and the continuity equation [Sabersky et al., 1989], it is possible in principle to compute the flow behavior with 
numerical methods. However, the computational complexity of this type of approach makes it ill-suited to use in developing a model that can be implemented on-line as part of a control law calculation, which is a requirement for the models being considered here.

The approach used here involves first, developing a model form that can describe the dominant flow physics; second, extending the model to 3-D using a strip-theory approach; and third, fitting key parameters within the model using experimental data. This method allows the dominant phenomena present in a particular flow situation to be modeled with accuracy and sufficient simplicity for on-line implementation.

\subsection{Model Form}

The first step in developing a model of the hydrodynamic forces that act on an underwater robotic manipulator is to develop a model form upon which a full three-dimensional model can be built. The procedure chosen for doing this begins with the development of a model for the case of two-dimensional, potential flow past a circular cylinder undergoing unsteady motions. In particular, the development presented by Sarpkaya [Sarpkaya, 1963, Sarpkaya and Garrison, 1963], which described a stationary cylinder in an accelerating flow, is extended to the case of an accelerating cylinder in a stationary fluid.

As stated in Equation 1, the principal result of this analysis is that the forces acting on a segment of an accelerating cylinder should be expressed with added mass and drag coefficients, $C_{m}$ and $C_{d}$, which are functions of distance traveled, instead of with the typical values $C_{d}=1.2$ and $C_{m}=1$.

Presented first are flow visualization results that motivate the $s / D$ dependence in the development of the hydrodynamic forces. This is followed by the two-dimensional, potential-flow-theory development.

\subsubsection{Experimental Flow Visualization}

Figure 3 shows flow visualization data as captured by a camera attached to the arm at the hub looking out toward the tip (i.e., down-the-arm). A patch of dye was applied to the trailing edge of the cylinder at a distance of $0.8 L$ out from the hub. For each image, the ratio $s / D$ gives the number of diameters the cylinder had traveled at the point where the dye was applied. From this perspective, the development of the wake as the cylinder rotates can be observed. This is useful since the drag forces on the cylinder are largely a function of the characteristics of the flow separation. In frame 1, it can be seen that initially the flow is completely attached; here the flow is potential in nature, and the hydrodynamic forces are due completely to fluid inertia and to skin friction (with skin friction being very small). As the cylinder travels, separation occurs and a pair of symmetric vortices begins to form in the near wake (frame 2). As the motion progresses, these vortices grow, and the separation points move to the top and bottom edges of the cylinder (frames 3 and 4). As the vortices grow stronger, pressure drag on the cylinder increases. The wake first widens (frame $5)$ and then narrows (frame 6) as the flow becomes fully established.

The key observation that can be made from these down-the-arm-view flow-visualization pictures is that the structure of the wake changes drastically as the cylinder accelerates from rest. Initially the flow is completely attached, but as the cylinder moves the flow develops to a state where it is fully separated. Because of this transition in the behavior of the flow, it is expected that the drag and added-mass coefficients will not remain constant, but will change to reflect the instantaneous characteristics of the flow.

\subsubsection{Theoretical Development}

The main assumptions of potential-flow theory are that the fluid is inviscid and the flow is irrotational. In regions outside the boundary layer and wake, these assumptions are completely valid. The challenge, though, in using a potential-flow analysis for a separated flow lies in correctly accounting for the effects of separation and the wake. In this analysis, a number of discrete vortices are used to model the wake created by the motion of the cylinder, which is justified based on the experimental flow-visualization photos showing the wake's development.

The analysis considers the forces acting on a cylinder that moves with unsteady motion through an incompressible, inviscid, nominally stationary fluid. Discrete vortices with independent positions, velocities, and strengths are used to model the feeding layers and wake induced by the cylinder motion. Figure 4 shows a schematic representation of the 2-D cylinder and its wake. The 2-D portion of this analysis is similar to 


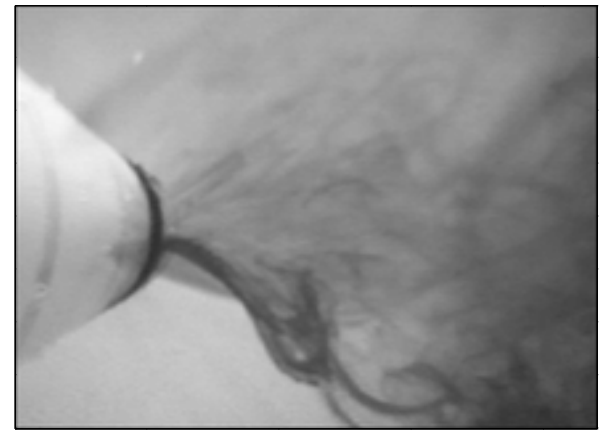

$\# 1:$ time $=0.50 \mathrm{sec}, \mathrm{s} / \mathrm{D}=1.2$

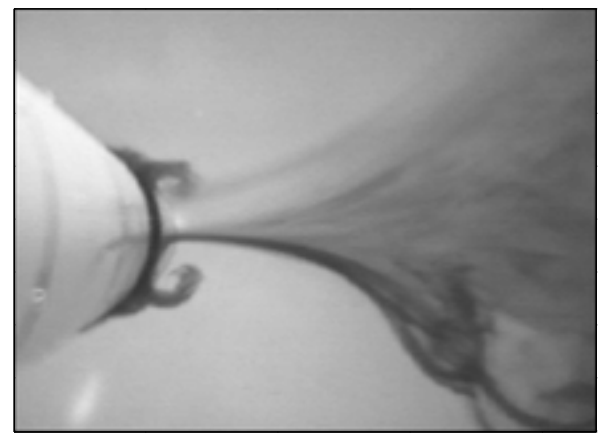

$\# 2:$ time $=0.83 \mathrm{sec}, \mathrm{s} / \mathrm{D}=1.7$

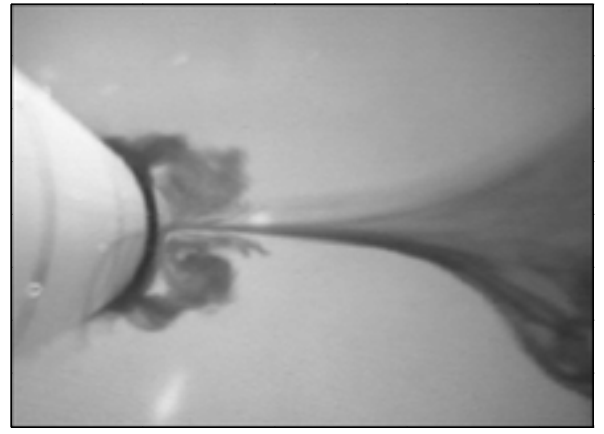

$\# 3:$ time $=1.03 \mathrm{sec}, \mathrm{s} / \mathrm{D}=2.3$

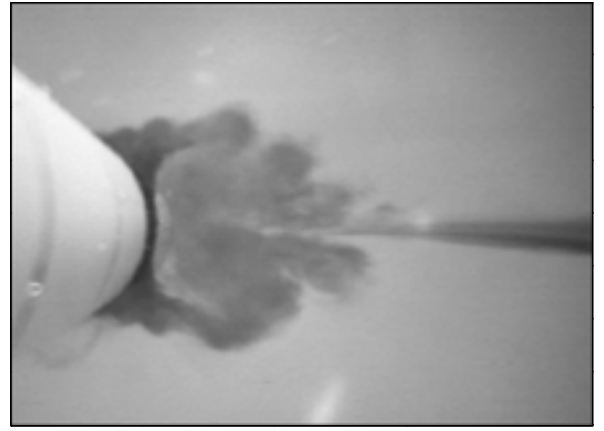

$\# 4:$ time $=1.33 \mathrm{sec}, \mathrm{s} / \mathrm{D}=3.5$

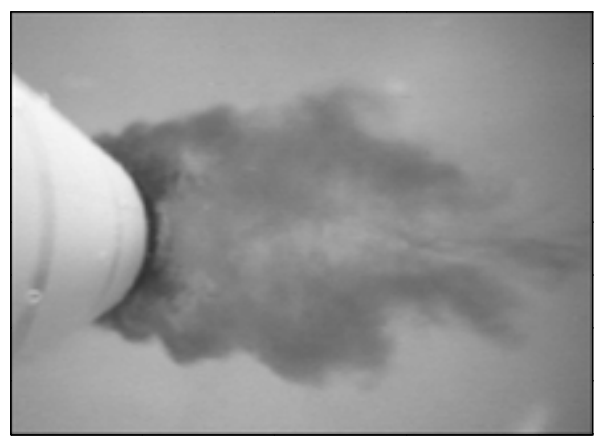

$\# 5:$ time $=1.67 \mathrm{sec}, \mathrm{s} / \mathrm{D}=5.2$

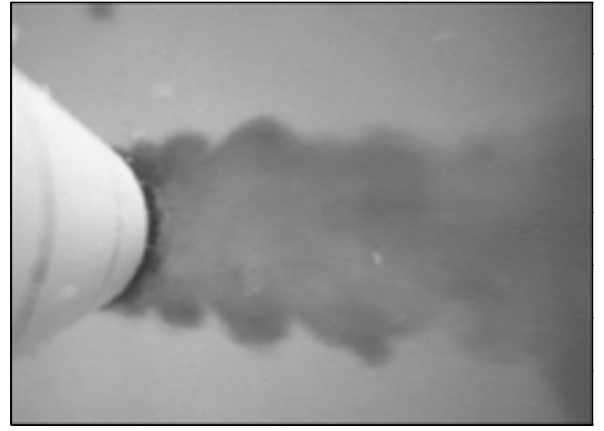

\#6: time $=2.27 \mathrm{sec}, \mathrm{s} / \mathrm{D}=8.6$

Figure 3: Flow Visualization - Down-the-Arm View

the approach of Sarpkaya [Sarpkaya, 1963, Sarpkaya and Garrison, 1963] for a stationary cylinder immersed in a moving fluid.

By extending Blasius' theorem [Milne-Thomson, 1960], the forces acting on a moving circular cylinder in a still fluid can be calculated by

$$
F_{X}-i F_{Y}=i \frac{\rho}{2} \int_{C}\left(\frac{d w}{d z}\right)^{2} d z-i \rho \frac{\partial}{\partial t} \int_{C} \bar{w} d \bar{z}+\rho A \frac{d \bar{W}}{d t}
$$

where $F_{X}$ and $F_{Y}$ represent drag and lift forces respectively, $w$ represents the complex velocity potential, $W$ represents the velocity of the reference frame fixed at the center of the cylinder, and $z$ denotes a complex variable. Each integral of Equation 2 is evaluated around the contour of the cylinder circumference $C$. For the 2-D model development, an overbar denotes the complex conjugate of a variable (e.g., $\bar{z}$ is the complex conjugate of $z$ ).

Using the circle theorem [Milne-Thomson, 1960], the complex velocity potential $w$, which describes the 


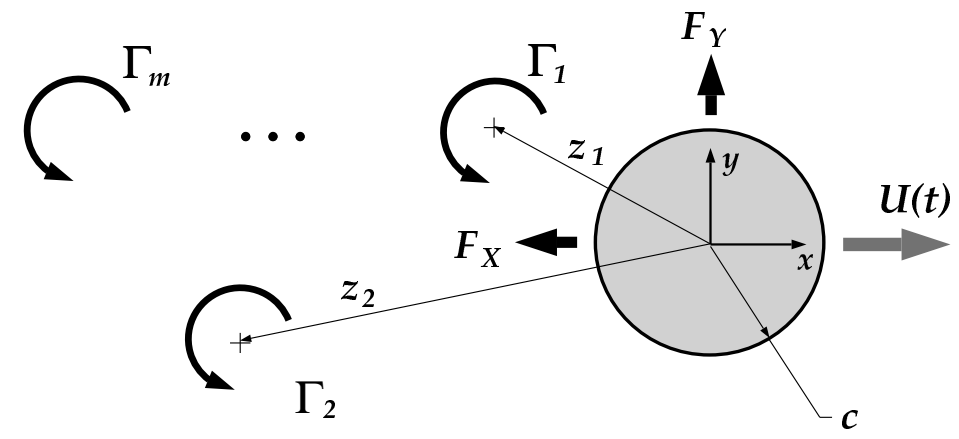

Figure 4: Two-Dimensional Hydrodynamic Model Schematic of Cylinder Moving Through Still Fluid

flow situation pictured in Figure 4, can be written as

$$
w=\frac{U(t) c^{2}}{z}+\frac{i}{2 \pi} \sum_{k=1}^{m} \Gamma_{k} \ln \left(z-z_{k}\right)-\frac{i}{2 \pi} \sum_{k=1}^{m} \Gamma_{k} \ln \left(z-\frac{c^{2}}{\bar{z}_{k}}\right)+\frac{i}{2 \pi} \sum_{k=1}^{m} \Gamma_{k} \ln z
$$

where $U(t)$ represents the unsteady cylinder velocity, $c$ represents the radius of the cylinder, and $\Gamma_{k}$ and $z_{k}$ represent the strength and position of the $k$ th vortex. While the last term of Equation 3 comes directly from the circle theorem, it should be excluded from the expression for $w$, since the vortices are generated by the relative motion of the cylinder with respect to the water. Because of this, the presence of the vortices does not change the net circulation around the cylinder, and it is not appropriate to include the image vortices at the center of the cylinder in the complex potential [Sarpkaya, 1968]. Therefore, Equation 3 can be rewritten as

$$
w=\frac{U(t) c^{2}}{z}+\frac{i}{2 \pi} \sum_{k=1}^{m} \Gamma_{k} \ln \left(z-z_{k}\right)-\frac{i}{2 \pi} \sum_{k=1}^{m} \Gamma_{k} \ln \left(z-\frac{c^{2}}{\bar{z}_{k}}\right)
$$

This expression is composed of a moving doublet term that models the cylinder, a term that models the $m$ real vortices in the feeding layers and wake, and a term that models the $m$ image vortices in the cylinder. The image vortices are included to satisfy the boundary conditions on the surface of the cylinder. Motion of the reference frame fixed at the center of the cylinder is accounted for by

$$
W=\bar{W}=U(t)
$$

where $W$ represents the velocity of the reference frame.

Equation 2 can be evaluated most easily by considering each term independently:

$$
\begin{aligned}
F_{X_{1}}-i F_{Y_{1}} & =i \frac{\rho}{2} \int_{C}\left(\frac{d w}{d z}\right)^{2} d z \\
F_{X_{2}}-i F_{Y_{2}} & =-i \rho \frac{\partial}{\partial t} \int_{C} \bar{w} d \bar{z} \\
F_{X_{3}} & =\rho A \frac{d \bar{W}}{d t}=\pi \rho c^{2} \frac{d U}{d t} .
\end{aligned}
$$

Equation 6 can be solved using Lagally's theorem [Milne-Thomson, 1960, Sarpkaya, 1963]. The steady part of the drag and lift forces that act on a cylinder with $m$ vortices alone with no circulation or stream present can be written as

$$
F_{X_{1}}-i F_{Y_{1}}=\rho \sum_{k=1}^{m} \Gamma_{k}\left(-v_{k}-i u_{k}\right)
$$

where $u_{k}$ and $v_{k}$ are real and imaginary components of the velocity of the center of the $k$ th real vortex. The integral on the right hand side of Equation 7 can be integrated by parts [Sarpkaya, 1968] to give

$$
F_{X_{2}}+i F_{Y_{2}}=-i \rho \frac{\partial}{\partial t} \int_{C} z \frac{d w}{d z} d z
$$


By using the residue theorem, the following expression for $F_{X_{2}}+i F_{Y_{2}}$ can be obtained:

$$
F_{X_{2}}+i F_{Y_{2}}=-2 \pi \rho c^{2} \frac{d U}{d t}-i \rho \sum_{k=1}^{m} \frac{c^{2}}{\bar{z}_{k}} \frac{\partial \Gamma_{k}}{\partial t}-i \rho \sum_{k=1}^{m} \Gamma_{k} \frac{\partial}{\partial t}\left(\frac{c^{2}}{\bar{z}_{k}}\right) \text {. }
$$

In this expression, the center position of the $k^{t h}$ image vortex, $c^{2} / \bar{z}_{k}$, can be written as

$$
\frac{c^{2}}{\bar{z}_{k}}=p_{k_{i}}+i q_{k_{i}}
$$

where $p_{k_{i}}$ and $q_{k_{i}}$ are the real and imaginary coordinates of the $k$ th image vortex position. The coordinates of the image vortices are related to the coordinates of the real vortices, $p_{k}$ and $q_{k}$, by the following expressions:

$$
\begin{aligned}
p_{k_{i}} & =\frac{p_{k} c^{2}}{p_{k}^{2}+q_{k}^{2}} \\
q_{k_{i}} & =\frac{q_{k} c^{2}}{p_{k}^{2}+q_{k}^{2}} .
\end{aligned}
$$

The velocity components of the image vortices, $u_{k_{i}}$ and $v_{k_{i}}$, are simply the partial derivatives with respect to time of each vortex coordinate:

$$
\begin{aligned}
& u_{k_{i}}=\frac{\partial p_{k_{i}}}{\partial t} \\
& v_{k_{i}}=\frac{\partial q_{k_{i}}}{\partial t}
\end{aligned}
$$

Finally, $F_{X_{2}}+i F_{Y_{2}}$ can be formulated as

$$
F_{X_{2}}+i F_{Y_{2}}=-2 \pi \rho c^{2} \frac{d U}{d t}+\rho \sum_{k=1}^{m} \frac{\partial \Gamma_{k}}{\partial t}\left(q_{k_{i}}-i p_{k_{i}}\right)+\rho \sum_{k=1}^{m} \Gamma_{k}\left(v_{k_{i}}-i u_{k_{i}}\right)
$$

Combining Equations 8, 9, and 17 and rearranging them into real and imaginary parts gives

$$
\begin{aligned}
& F_{X}=-\rho \sum_{k=1}^{m} \Gamma_{k}\left(v_{k}-v_{k_{i}}\right)+\rho \sum_{k=1}^{m} q_{k_{i}} \frac{\partial \Gamma_{k}}{\partial t}-\frac{\pi}{4} \rho D^{2} \frac{d U}{d t} \\
& F_{Y}=\rho \sum_{k=1}^{m} \Gamma_{k}\left(u_{k}-u_{k_{i}}\right)-\rho \sum_{k=1}^{m} p_{k_{i}} \frac{\partial \Gamma_{k}}{\partial t}
\end{aligned}
$$

The remainder of the analysis focuses on the in-line force term $F_{X}$. Based on the underlying physics involved, it is evident that the strengths of vortices in the trailing wake depend on the characteristics of the cylinder, fluid, and motion. For constant-acceleration motion, $\Gamma_{k}$ can be expressed as a function of the diameter, velocity, and acceleration of the cylinder; the density and kinematic viscosity of the fluid; and time:

$$
\Gamma_{k}=\mathcal{F}_{1}\left(D, U, \frac{d U}{d t}, \rho, \nu, t\right)
$$

A dimensional analysis of these variables shows that

$$
\frac{\Gamma_{k}}{U D}=\mathcal{F}_{2}\left(\frac{\nu}{U D}, \frac{U t}{D}\right)=\mathcal{F}_{3}(R e, s / D)
$$

where $s$ is the displacement of the cylinder, $D$ is the diameter of the cylinder, and $R e$ is the Reynolds number. Using the same approach, it can be shown that

$$
\frac{u_{k}}{U}, \frac{v_{k}}{U}, \frac{u_{k i}}{U}, \frac{v_{k i}}{U}, \frac{p_{k}}{D}, \frac{q_{k}}{D}, \frac{p_{k i}}{D}, \frac{q_{k i}}{D} \text { are functions of } \frac{s}{D} \text { and } R e .
$$


Experiments conducted by Sarpkaya and Garrison [Sarpkaya and Garrison, 1963] found that over a wide range of Reynolds numbers $\left(100<R e<5 \times 10^{5}\right)$ the above dimensionless parameters have only a strong functional dependence on $s / D$. Accordingly, letting

$$
\frac{\Gamma_{k}}{U D}=\mathcal{G}(s / D)
$$

and differentiating $\Gamma_{k}$ with respect to time gives

$$
\frac{\partial \Gamma_{k}}{\partial t}=D \frac{\Gamma_{k}}{U D} \frac{d U}{d t}+\frac{\partial\left(\Gamma_{k} / U D\right)}{\partial(s / D)} U^{2} .
$$

By substituting the results from Equation 23 into Equation 18, the following result is obtained:

$$
\begin{aligned}
F_{X}= & -\frac{\pi}{4} \rho D^{2} \frac{d U}{d t}\left[1-\sum_{k=1}^{m} \frac{4}{\pi} \frac{q_{k_{i}}}{D} \frac{\Gamma_{k}}{U D}\right] \\
& -\frac{1}{2} \rho D U^{2}\left[2 \sum_{k=1}^{m} \frac{\Gamma_{k}}{U D}\left(\frac{v_{k}}{U}-\frac{v_{k_{i}}}{U}\right)-2 \sum_{k=1}^{m} \frac{q_{k_{i}}}{D} \frac{\partial\left(\Gamma_{k} / U D\right)}{\partial(s / D)}\right] .
\end{aligned}
$$

For constant-acceleration motions, the bracketed expressions in Equation 24 are functions of $s / D$ only. Therefore, $F_{X}$ can be expressed as

$$
F_{X}=-C_{m}(s / D) \cdot \frac{\pi}{4} \rho D^{2} \frac{d U}{d t}-C_{d}(s / D) \cdot \frac{1}{2} \rho D U^{2}
$$

where

$$
\begin{aligned}
C_{m}(s / D) & =1-\sum_{k=1}^{m} \frac{4}{\pi} \frac{q_{k_{i}}}{D} \frac{\Gamma_{k}}{U D} \\
C_{d}(s / D) & =2 \sum_{k=1}^{m} \frac{\Gamma_{k}}{U D}\left(\frac{v_{k}}{U}-\frac{v_{k_{i}}}{U}\right)-2 \sum_{k=1}^{m} \frac{q_{k_{i}}}{D} \frac{\partial\left(\Gamma_{k} / U D\right)}{\partial(s / D)} .
\end{aligned}
$$

Equation 25 is the key result. It states that for a cylinder undergoing constant acceleration motions (relative to nominally still water), the hydrodynamic drag and added-mass coefficients, $C_{d}$ and $C_{m}$, are functions of how far the cylinder has traveled only. In other words, the instantaneous values of $C_{d}$ and $C_{m}$ are independent of the cylinder's acceleration, its velocity, or the associated Reynolds number and are dependent only on the cylinder's displacement from its initial resting position.

\subsection{Strip-Theory Extension to 3-D}

Using a strip-theory approach, the results of the above 2-D analysis can be extended semi-empirically to three dimensions. This approach is diagramed in Figure 5. The forces acting on a thin segment of the arm are calculated using a form of Equation 25:

$$
d F_{i}=-C_{m_{i}}\left(l_{i} \theta / D\right) \cdot \rho \frac{\pi D^{2}}{4} l_{i} d l_{i} \ddot{\theta}-C_{d_{i}}\left(l_{i} \theta / D\right) \cdot \frac{1}{2} \rho D l_{i}^{2} d l_{i}|\dot{\theta}| \dot{\theta} .
$$

where the distance traveled of the $i^{t h}$ segment is due (in this case) solely to rotation about the hub: $s_{i}=l_{i} \theta$ and $U_{i}=l_{i} \dot{\theta}$.

Given this, a standard strip-theory approach allows the hydrodynamic in-line torque and force acting at the hub to be calculated with the following simple relations:

$$
\begin{array}{r}
d T_{i}=l_{i} d F_{i} \\
T_{h y d}=\sum_{i=1}^{n} d T_{i} \\
F_{\text {hyd }}=\sum_{i=1}^{n} d F_{i}
\end{array}
$$




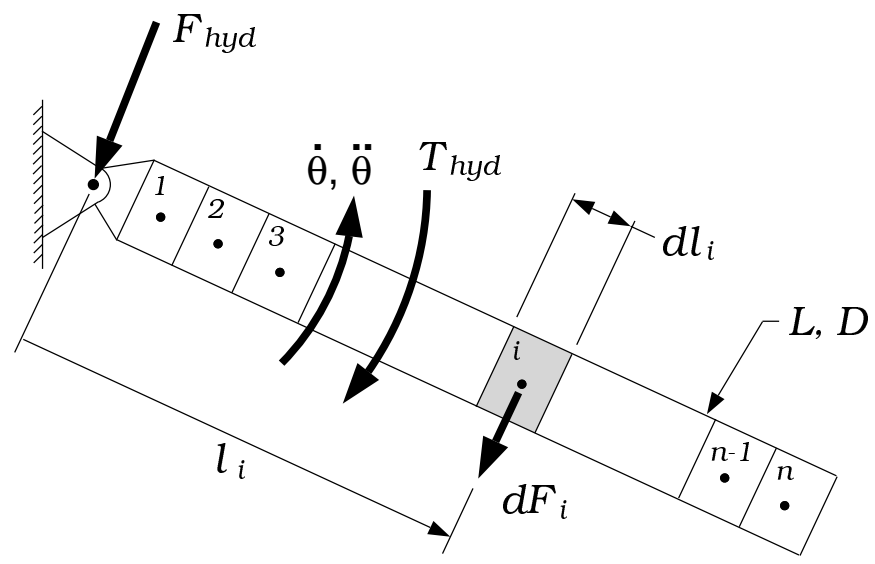

Figure 5: Diagram of Strip-Theory Implementation

where $n$ is the number of segments used in the model.

Finally, if the assumption is made that the functional relationships that describe the added-mass and drag coefficients for each individual segment $\left(C_{m_{i}}\left(l_{i} \theta / D\right)\right.$ and $\left.C_{d i}\left(l_{i} \theta / D\right)\right)$ can be replaced by global "average" coefficient relationships, then Equation 30 can be rewritten as

$$
T_{h y d}=-\sum_{i=1}^{n}\left(\bar{C}_{m}\left(l_{i} \theta / D\right) \cdot \rho \frac{\pi D^{2}}{4} l_{i}^{2} d l_{i} \ddot{\theta}+\bar{C}_{d}\left(l_{i} \theta / D\right) \cdot \frac{1}{2} \rho D l_{i}^{3} d l_{i}|\dot{\theta}| \dot{\theta}\right) .
$$

where $\bar{C}_{d}$ and $\bar{C}_{m}$ have been introduced to indicate these average coefficient functions.

\subsection{Empirical Determination of Hydrodynamic Coefficients}

Given the relationship of Equation 32, and the hub torque, angular position, velocity, and acceleration measurements from the single-link arm, the remaining step is to determine how the hydrodynamic coefficients, $\bar{C}_{d}$ and $\bar{C}_{m}$, vary with respect to the travel or displacement $(s / D)$ of the cylinder. This is accomplished using parameter identification. The primary advantage of using global average coefficient functions is that only two functions have to be identified for the whole arm rather than two functions for each segment used in the model; specific configuration and 3-D effects (e.g., end effects, etc.) are approximated as a lumped effect within the identified coefficients. A disadvantage is that specific information about the local coefficients is not captured.

Hydrodynamic-Coefficient Identification Method Equation 32 expresses the relationship between the hydrodynamic torque acting and the angular displacement, velocity, and acceleration of the arm - all measured quantities in this research. The unknown parameters of interest are the hydrodynamic drag and added-mass coefficients. The primary challenge in characterizing these coefficients is that for a particular instant in time, Equation 32 is underdetermined - that is, there are two unknown quantities $\left(\bar{C}_{d}\right.$ and $\left.\bar{C}_{m}\right)$ and only one expression to relate them.

To overcome this difficulty, an approach for identifying $\bar{C}_{d}$ and $\bar{C}_{m}$ was developed. This approach models $\bar{C}_{d}$ and $\bar{C}_{m}$ as a series of cubic-spline polynomials that are functions of $s / D$. Nonlinear optimization was used to determine the locations of the spline points, and hence the shape of the polynomial functions that model the hydrodynamic coefficients. A unique feature of this approach is that it incorporates previously gained knowledge of the hydrodynamics into the coefficient profiles.

Figure 6 illustrates the polynomial spline models used for the drag and added-mass coefficients prior to fitting to the experimental data. Each curve is composed of three types of spline points: one fixed point, five shaping points, and one scaling point. For both coefficients, the fixed points correspond to the start-ofmotion values of the coefficients predicted by the potential-flow theory; in the initial instants of motion, the 
value of $\bar{C}_{d}$ is fixed at 0 , and the value of $\bar{C}_{m}$ is fixed at 1 . These two points remain unchanged during the optimization process.

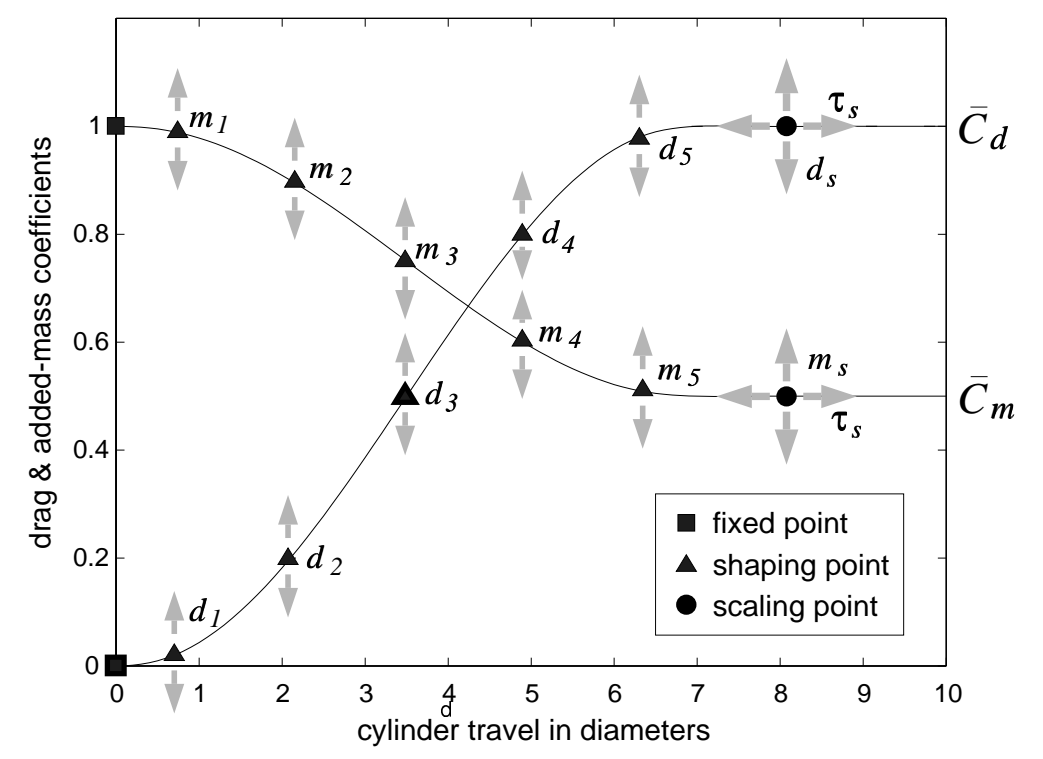

Figure 6: Drag and Added-Mass Coefficient Spline Models

For each coefficient, five equally-spaced shaping points are used to vary the shape of the coefficient profile. Movement of these shaping points allows the coefficient curves to take on a wide variety of shapes that properly model the state-dependent behavior of the coefficients.

Each coefficient curve is scaled both in the travel dimension and the coefficient-magnitude dimension by a single scaling point. Movement of this point adjusts the scale of the entire coefficient profile between the fixed and scaling points. Location of this scaling point effectively determines the steady-state value of the coefficient and the travel value after which the coefficient is assumed to be constant.

Given measurements of arm position, velocity, acceleration, and hub torque $\left(T_{\text {meas }}\right)$, the nonlinear optimization problem of fitting the hydrodynamic coefficients to the experimental torque data can be posed as follows:

Find $\left(d_{1}, d_{2}, d_{3}, d_{4}, d_{5}, d_{s}, m_{1}, m_{2}, m_{3}, m_{4}, m_{5}, m_{s}, \tau_{s}\right)$

$$
\text { to minimize } \sum_{i=1}^{m}\left(T_{\text {hyd }_{i}}-T_{\text {meas }_{i}}\right)^{2}
$$

where $T_{h y d}$ comes from Equation 32 and $m$ is the number of data points being considered. For the coefficient data presented below, a total of 12 different arm slews at four different accelerations were used to evaluate the coefficients. For each of the 12 data sets, measurements of hub torque,position, velocity, and acceleration at 200 to 500 time points (depending on the duration of the slew) were used to determine the best-fit location of the coefficient spline points. The identification of one coefficient profile for multiple runs of different accelerations is in accordance with the theoretically derived dependence of the coefficients on travel only.

Modeling the coefficients as cubic splines and then using nonlinear optimization to vary the shape of the splines allows two parameters to be determined simultaneously from one equation by considering not just each instant in time individually, but by optimizing the coefficient profile shapes (using a relatively small number of parameters) over their entire transients. This approach also allows previously determined physical information about the coefficients' behavior to be included in the parameterization of their profiles.

Results: Experimentally Identified Coefficients Figure 7 shows values for the drag and added-mass coefficients that were obtained using the optimization procedure described above. The drag coefficient initially starts off at zero as constrained by the fixed spline point. The drag coefficient rises rapidly and peaks at a value just larger than 1.0 after about 3.7 diameters of travel. The drag coefficient then drops off 
and settles to a steady-state value of 0.78 after 8 diameters of motion. The added-mass coefficient starts off initially at a value of 1.0 and drops off to a value of 0.2 after 4.2 diameters of motion. The added-mass coefficient then rises to its steady-state value of 0.36 .

Variation of the coefficients for different slews is indicated by the data points marked with + symbols. These points were determined by performing the optimization procedure on three groups of data from four different slews (rather than one group of data from 12 slews).

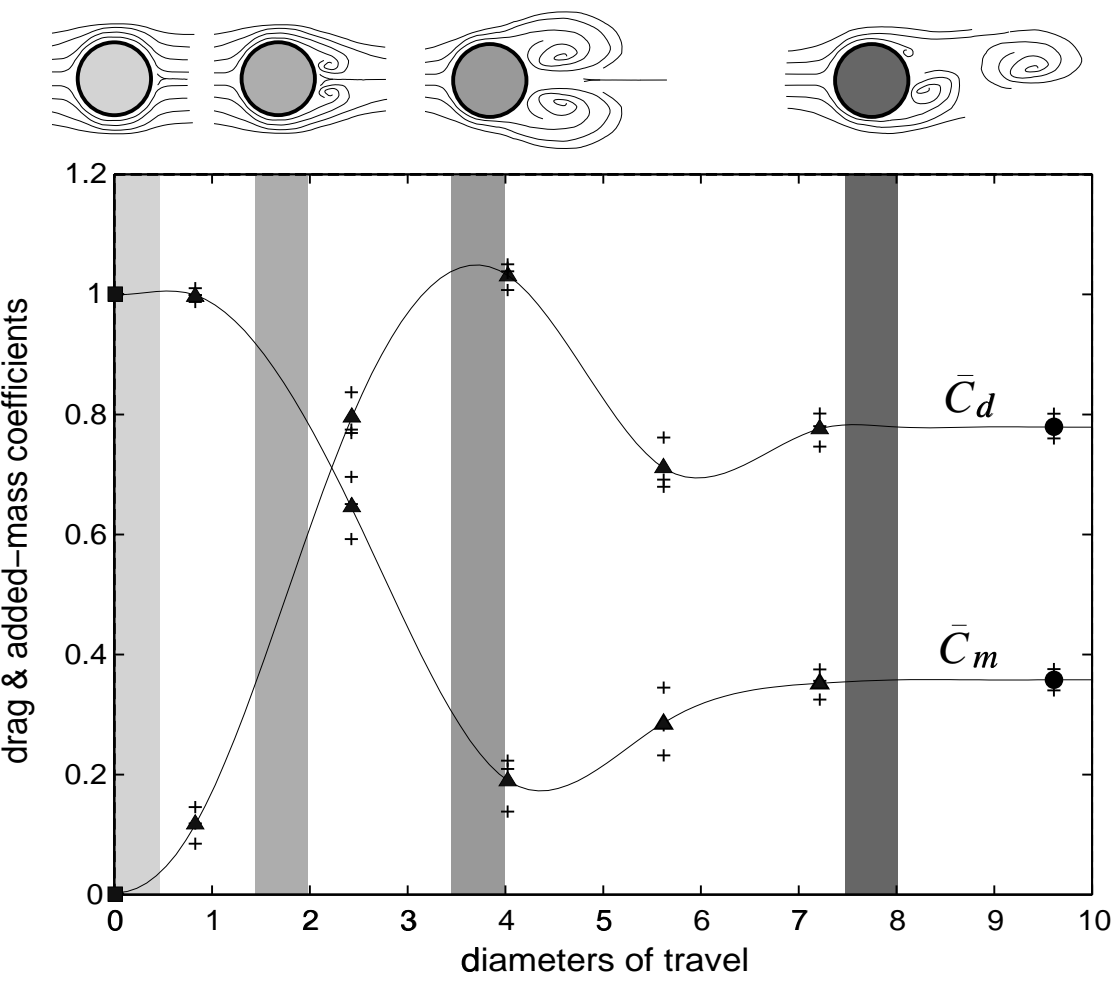

Figure 7: Drag and Added-Mass Torque Coefficients

It is important to note that these results correlate well with those from both the flow visualization and the 2-D theoretical analysis. For $s / D=0$, the drag and added-mass coefficient values are fixed at 0 and 1 respectively, as potential-flow theory predicts for the case of fully attached flow. As the flow separates and a symmetric vortex pair begins to form in the near wake, the drag coefficient rises. The drag coefficient reaches its peak when the vortices are at their largest size, which is just prior to their being shed. When the symmetric vortices can no longer coexist in a stable manner in the near wake, vortex shedding begins. When this happens, the drag coefficient drops off, then it rises to its steady-state value that corresponds to the fully developed wake.

Equation 27 shows the dependence of the drag coefficient on the development of vortices in the wake: When no vortices are present, the drag coefficient is zero; as vortices grow and move in the wake, the drag coefficient rises accordingly.

Because of the three-dimensional flows along the span and around the tip, the steady-state drag coefficient (0.78) is considerably lower than the value of 1.2 expected for a translating cylinder with no end effects. Flow around the tip and along the span relieve the low-pressure area at the base (trailing side) of the arm near the tip, which results in lower drag forces and hence a lower mean-drag coefficient. Furthermore, acceleration of the arm promotes attachment of the flow, which results in slightly lower values of $\bar{C}_{d}$ than for constant velocity motions.

From the behavior of the added-mass coefficient, it can be concluded that the presence of vortices in the near wake reduces the amount of fluid that is accelerated locally as the cylinder is accelerated. This result agrees completely with the two-dimensional potential-flow-theory analysis results shown in Equation 26: With no vortices in the wake, $\bar{C}_{m}=1$; as separation occurs and vortices grow in the wake, the added-mass 
coefficient drops off from this initial value. As long as separation occurs and vorticity is shed into the wake, it is expected that the added-mass coefficient will be smaller than the value of 1 , which is predicted by potential-flow theory (without separation effects).

\section{$3.4 \quad 3-D$ Effects}

The above analysis dealt with the effects of 3-D flow only approximately. In particular, the development of Equation 32 involved an implicit assumption that the $C_{m_{i}}$ and the $C_{d_{i}}$, although modified by 3 -D effects, were the same for each segment along the arm. The utility of this assumption will be demonstrated in the following section, in which it is shown that the resulting model is able to achieve excellent results in describing the hydrodynamic forces that act on the arm.

However, it should be noted that the underlying assumption is only an approximation. For example, the flow visualization results presented in Figure 8 demonstrate several features. In the first frame, the flow is attached initially over the full surface of the cylinder. As the motion progresses, there is evidence of separation along the trailing edge of the arm (frame 2). The line of separation moves from the trailing edge to the top of the cylinder as it rotates (frames 2-5). Along the leading edge, prior to separation, the flow is normal to the arm. Flow separation is most evident from the span-wise flow radially outward that occurs after the flow separates. The flow along the trailing side (base) of the arm from the hub toward the tip is caused by the pressure differential between the near-ambient base pressure at the hub and the lower base pressure near the tip. There is also flow around the tip of the cylinder from the ambient-pressure region beyond the tip to the lower-base-pressure area just inside the tip.

The most significant observation from the top-view flow visualization data is that the flow over a rotating circular cylinder is highly three-dimensional. Flow along the span and around the tip of the arm relieve lowpressure areas along the trailing side of the arm, which are the main source of drag. Because of the 3-D flow, lower-than-normal drag-coefficient values are expected than for a cylinder undergoing purely translational motion. This is accounted for in the identification of $\bar{C}_{m}$ and $\bar{C}_{d}$ as described above. Also expected however, is a variation in the effective coefficients along the length of the arm because of three-dimensional effects that occur locally. This effect could be accounted for by adding additional free parameters to the parameter identification approach, but this has not been done here (largely because of the success achieved without the added complexity).

\section{Experimental Model Validation}

This section presents experimental results that validate the accuracy of the model developed in the previous section. For the results presented here, the arm was divided into 10 equal segments. This hydrodynamic model is relatively undemanding from a computational point of view and has been implemented at sample rates up to $500 \mathrm{~Hz}$ on a 68030-based real-time processor using four segments.

Figures 9 through 11 compare modeled hydrodynamic torque predictions from the model developed here and two other models from the literature to the actual hydrodynamic torques that were measured experimentally using the shaft-torque sensor located at the hub of the arm. Figure 9 shows results from a short 30-degree, 0.4-second slew; Figure 10 shows results from a 60-degree, 0.6-second slew; and Figure 11 shows results from a longer 120-degree, 1.2-second slew. Results from three different slews demonstrate that the model developed here is valid for a wide range of motions. In each figure, plot (a) shows the time history of the arm motion. Plot (b) shows results from the model developed as part of this research that uses state-dependent coefficients. Plot (c) shows results from a model implemented according to Lévesque and Richard [Lévesque and Richard, 1994] wherein the effects of added-mass were not considered, and the drag coefficient was assumed to a have a constant value of 1.1. Plot (d) shows results from the model in which the drag coefficient had a constant value of 1.2 , and the added-mass coefficient had a constant value of 1 .

The results of Figures 9 through 11 demonstrate the significant improvement in modeling accuracy obtained using the approach of this paper compared to other methods presented in the literature. On average, modeling errors ${ }^{1}$ were reduced by 4.5 times when comparing the model of this research to the other

\footnotetext{
${ }^{1}$ Model errors were calculated by normalizing the sum of the errors for the data sets presented in Figures 9-11 according to
} 


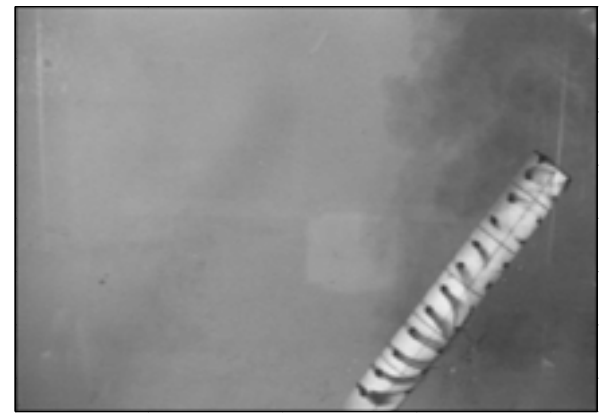

$\# 1:$ time $=0.43 \mathrm{sec}$

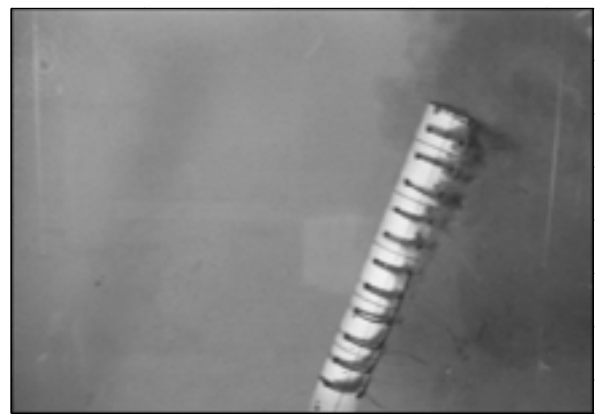

$\# 2:$ time $=1.23 \mathrm{sec}$

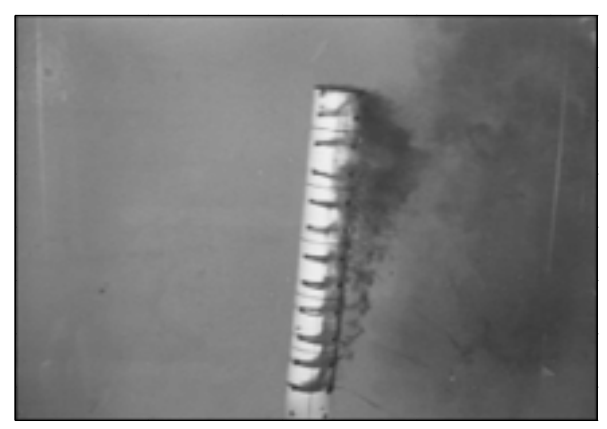

\#3: time $=1.70 \mathrm{sec}$

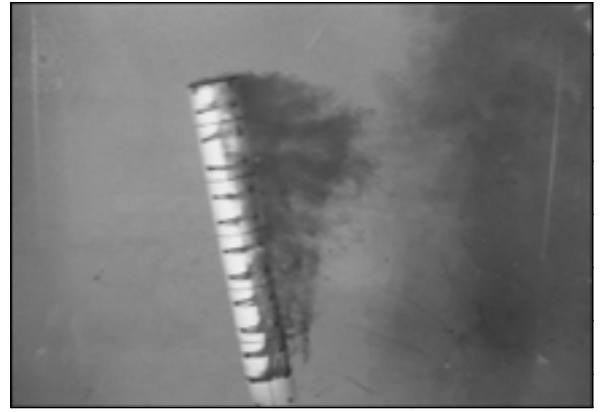

\#4: time $=2.06 \mathrm{sec}$

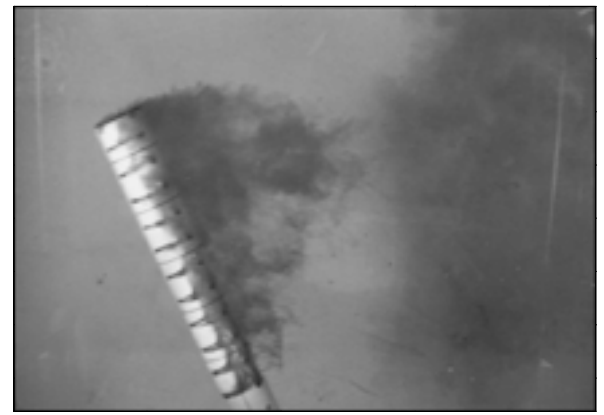

\#5: time $=2.33 \mathrm{sec}$

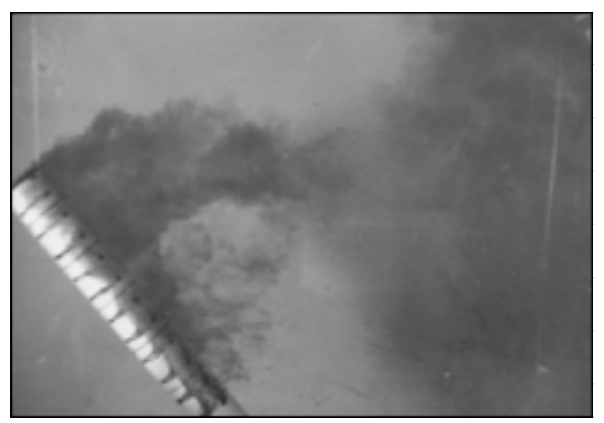

\#6: time $=2.60 \mathrm{sec}$

Figure 8: Flow Visualization - Top View

two models considered. This higher level of accuracy was achieved by using state-dependent drag and addedmass coefficients. During initial portions of the slews, these coefficients take on values that correspond to values predicted by potential flow theory $\left(\bar{C}_{d}=0\right.$ and $\left.\bar{C}_{m}=1\right)$. As the motion progresses, the coefficients transition to take on steady-state values that correspond to the three-dimensional separated flow situation $\left(\bar{C}_{d}=0.78\right.$ and $\left.\bar{C}_{m}=0.36\right)$.

In plot (c) of Figures 9, 10, and 11, it can be seen that this constant-coefficient model fails to capture the effects of the inertial torques, which dominate the beginning and end of the slew; and that it overestimates the drag during the middle portion of the slew. In the results of plot (d), this constant-coefficient model initially models the inertial torques well, but it later overestimates both the inertial and drag torque components. The major sources of error in the models presented in plots (c) and (d) are caused by the assumption that the coefficients are constant and that the drag coefficients have the same values as those that correspond to the translational motion of a cylinder $\left(\bar{C}_{d}=1.1-1.2\right)$; they do not, however, because of the radial flow induced by the swinging motion. In other words, the errors can be attributed to using coefficients from a

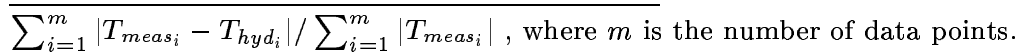


(a) 30 degree/ 0.4 second slew

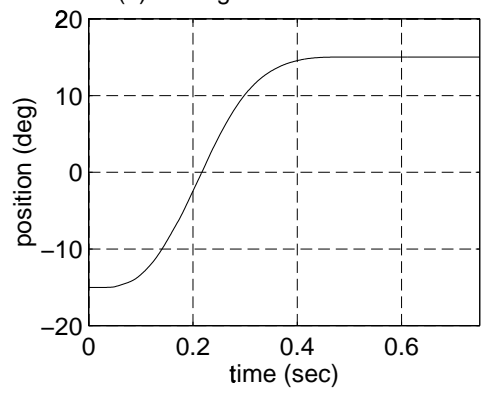

(c) $\overline{\mathrm{C}} \mathrm{d}=1.1, \overline{\mathrm{C}} \mathrm{m}=0$

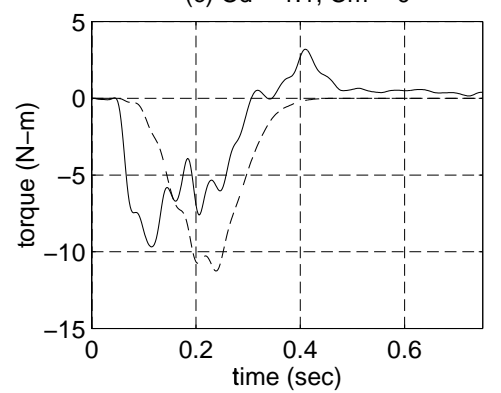

(b) state-dependent $\bar{C} d, \bar{C} m$

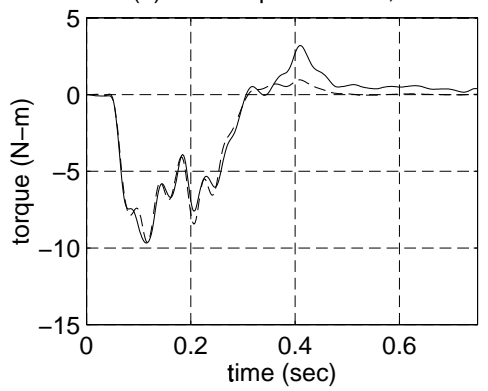

(d) $\bar{C} d=1.2, \bar{C} m=1$

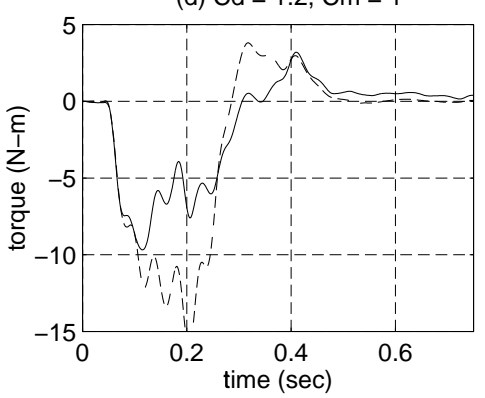

actual

- - - model

Figure 9: Hydrodynamic Modeling Results — Short Motion

(a) 60 degree/ 0.6 second slew

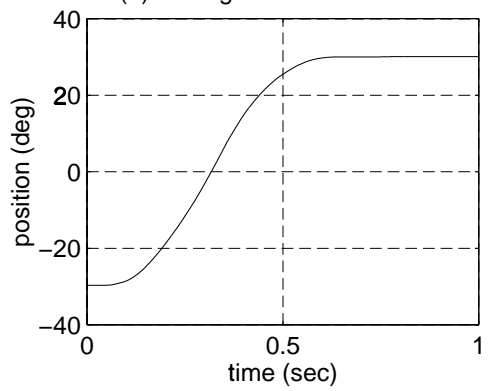

(c) $\overline{\mathrm{C}} \mathrm{d}=1.1, \overline{\mathrm{C}} \mathrm{m}=0$

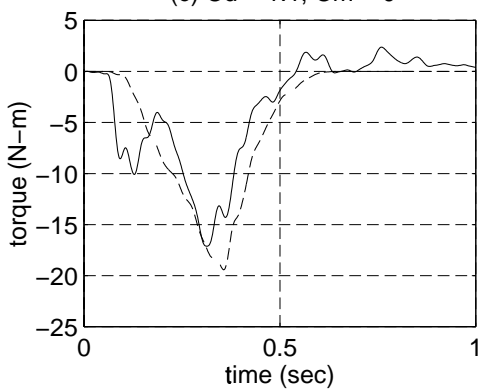

(b) state-dependent $\bar{C} d, \bar{C} m$

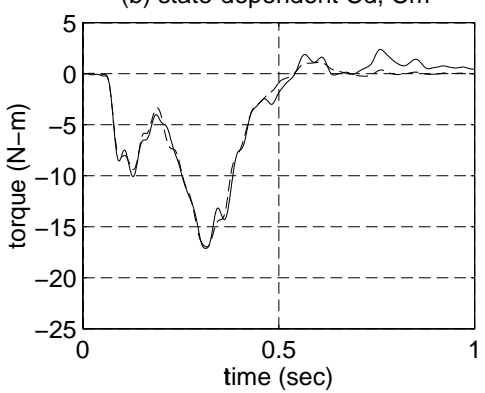

(d) $\bar{C} d=1.2, \bar{C} m=1$

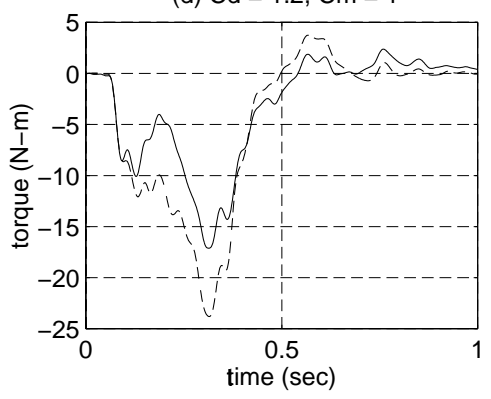

_ actual _-.. model

Figure 10: Hydrodynamic Modeling Results — Medium-length Motion

two-dimensional, steady-flow situation to describe a three-dimensional, unsteady flow.

While the data presented in Figures 9 through 11 are for constant-acceleration/constant-deceleration motions, it should be noted that the model developed here has been applied with equal success to fifthorder-spline trajectories (where acceleration is not constant), which are common for robotic manipulators. 
(a) 120 degree/1.2 second slew

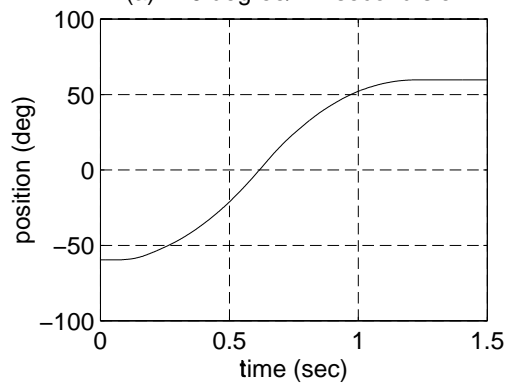

(c) $\overline{\mathrm{C}} \mathrm{d}=1.1, \overline{\mathrm{C}} \mathrm{m}=0$

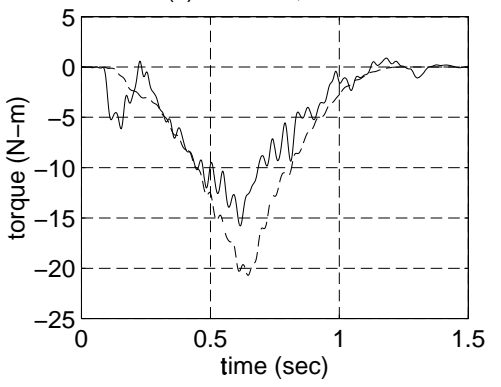

(b) state-dependent $\bar{C} d, \bar{C} m$

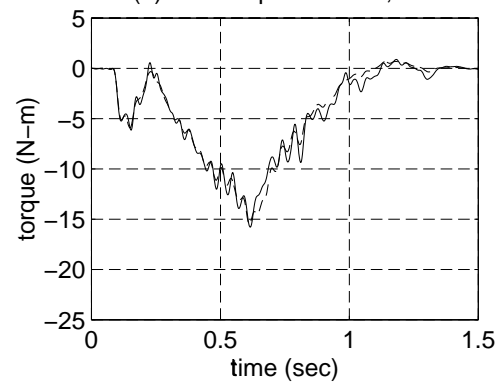

(d) $\bar{C} d=1.2, \bar{C} m=1$

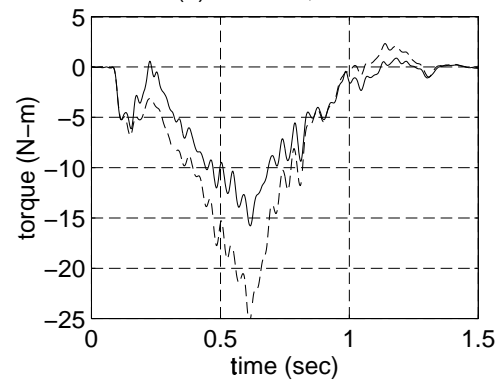

actual

-- - model

Figure 11: Hydrodynamic Modeling Results — Long Motion

\section{Implementation Issues}

Model Accuracy Versus Number of Segments A reasonable expectation is that the hydrodynamic model's accuracy will increase with the number of segments used to model the arm. Figure 12, which shows the modeling error plotted against the number of model segments used, confirms this expectation. Interestingly, the modeling error does not decrease significantly when more than four model segments are used. These results demonstrate that using a large number of segments, which increases the computational requirements, is not necessary to achieve accurate results using this improved model.

Drag Versus L/D Ratio The forces that act on a cylinder are dependent wholly on the behavior of the flow. For the rotating single link considered here, the flow is three-dimensional. The three-dimensional characteristics of the flow depend on the arm's geometry, primarily its length-to-diameter ratio $(L / D)$. The parameter most affected by changes in $L / D$ is the drag coefficient.

Figure 13 illustrates the effect of the $L / D$ ratio on the average drag coefficient determined from constantvelocity motions of the arm. These drag coefficients were determined directly from measurements of joint torque and joint-angle velocity using the strip-model approach of Equation 32 with $\bar{C}_{d}$ constant and the added-mass portion of the equation set to zero.

It can be seen that the effective drag coefficient increases with $L / D$. This is because for arms with high $L / D$, end-effects act over a smaller percentage of the span and the motion becomes more translational near the tip of a high- $L / D$ arm than that of a low- $L / D$ arm. In essence, 3-D flow effects become less significant as $L / D$ is increased. It is expected that for extremely high $L / D$ ratios (beyond what is feasible for manipulators), the drag coefficient will approach values typical for a translating cylinder $\left(\bar{C}_{d}=1.2\right)$.

\section{Conclusions}

In this paper, a detailed analysis of the hydrodynamic forces and torques that act on underwater manipulators has been presented. The short, accelerative, highly rotational motions and end effects associated with robotic manipulators result in three-dimensional transient flows. These complex flows over the arm generate large 


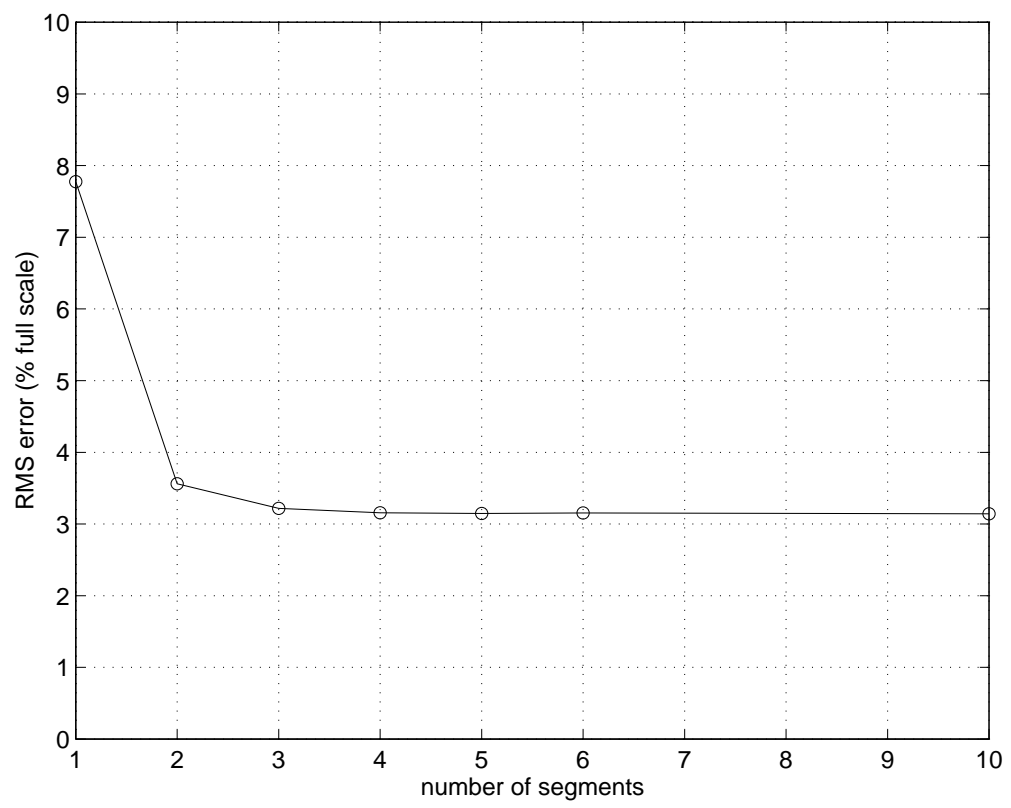

Figure 12: Modeling Error Versus Number of Segments

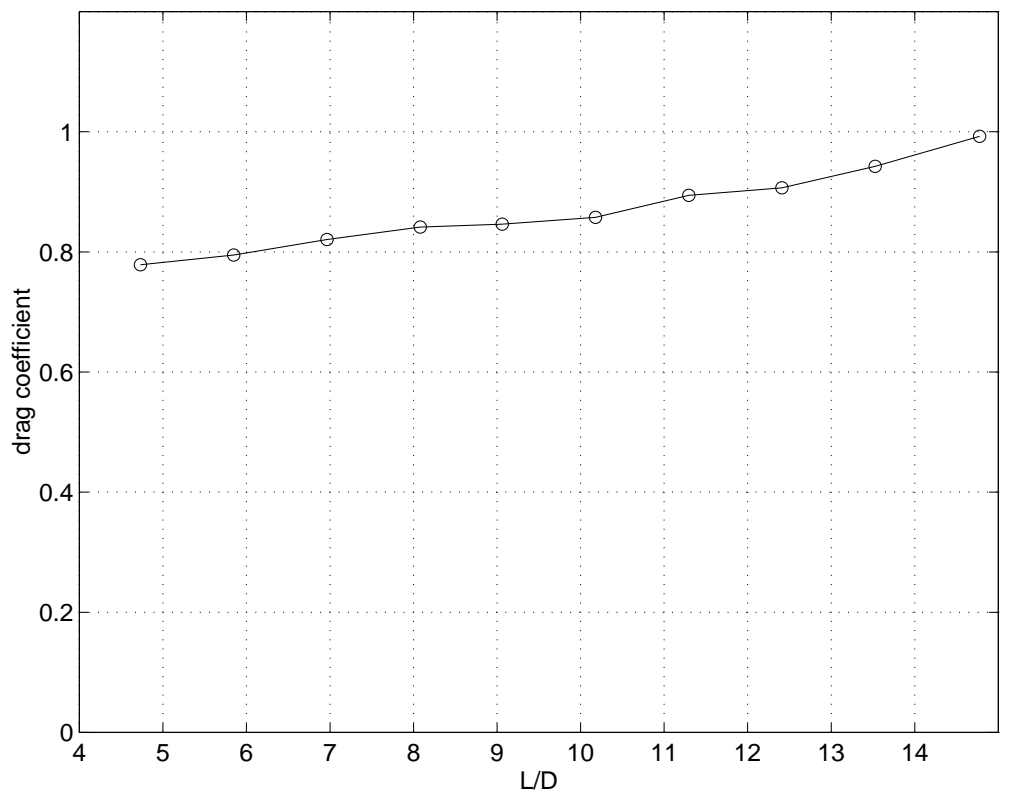

Figure 13: Drag Coefficient Versus L/D Ratio

hydrodynamic forces (relative to the inertial forces involved) that are difficult to model accurately. An accurate, real-time implementable model of the hydrodynamic in-line forces that act on a swinging circular cylinder has been formulated. The form of the model was developed beginning with a two-dimensional analysis of a circular cylinder that moved unsteadily in an incompressible, inviscid, and stationary fluid; discrete vortices trailed the cylinder to model the feeding layers and wake. This model was extended to the three-dimensional regime using strip theory. Added-mass and drag coefficients were identified from position, velocity, acceleration, and torque measurements using an approach developed for this work. Results showed that these coefficients are not constant but are rather state-dependent functions of how far the cylinder has traveled. The behavior of the experimentally obtained drag and added-mass coefficient profiles 
is well-supported by the flow-visualization results and the results of the two-dimensional theoretical analysis presented. Experimental results showed a significant improvement in hydrodynamic modeling accuracy when state-dependent drag and added-mass coefficients are used.

\section{Acknowledgments}

This research was supported by ONR Contract N0014-92-J-1943, NASA Contract NCC 2-333, and the Monterey Bay Aquarium Research Institute.

\section{References}

[Lévesque and Richard, 1994] Benoît Lévesque and Marc J. Richard. Dynamic analysis of a manipulator in a fluid environment. International Journal of Robotics Research, 13(3):221-231, 1994.

[McLain, 1995] Timothy W. McLain. Modeling of Underwater Manipulator Hydrodynamics with Application to the Coordinated Control of an Arm/Vehicle System. PhD thesis, Stanford University, August 1995. Also published as SUDAAR 670.

[McMillan et al., 1995] Scott McMillan, David E. Orin, and Robert B. McGhee. Efficient dynamic simulation of an underwater vehicle with a robotic manipulator. IEEE Transactions on Systems, Man, and Cybernetics, 25(8):1194-1206, 1995.

[Milne-Thomson, 1960] L. M. Milne-Thomson. Theoretical Hydrodynamics. The MacMillan Company, 4th edition, 1960.

[Obasaju et al., 1988] E.D. Obasaju, P.W. Bearman, and J.M.R. Graham. A study of forces, circulation, and vortex patterns around a cylinder in oscillating flow. Journal of Fluid Mechanics, 196:467-494, 1988.

[Sabersky et al., 1989] Rolf H. Sabersky, Allan J. Acosta, and Edward G. Hauptmann. Fluid Flow: A First Course in Fluid Mechanics. Macmillan, 3rd edition, 1989.

[Sarpkaya and Garrison, 1963] Turgut Sarpkaya and C. J. Garrison. Vortex formation and resistance in unsteady flow. Journal of Applied Mechanics, pages 16-24, March 1963.

[Sarpkaya, 1963] Turgut Sarpkaya. Lift, drag, and added-mass coefficients for a circular cylinder immersed in a time-dependent flow. Journal of Applied Mechanics, pages 13-15, March 1963.

[Sarpkaya, 1968] Turgut Sarpkaya. An analytical study of separated flow about circular cylinders. ASME Journal of Basic Engineering, 90:511-520, 1968.

[Sarpkaya, 1992] Turgut Sarpkaya. Brief reviews of some time-dependent flows. ASME Journal of Fluids Engineering, 114(3):283-298, September 1992.

[Tarn et al., 1996] T.J. Tarn, G.A. Shoults, and S.P. Yang. Dynamic modelling of an underwater vehicle with a robotic manipulator using Kane's method. Autonomous Robots, 3:269-283, 1996. 